Nervenarzt 2012 $\cdot 83: 1531-1532$

DOI 10.1007/s00115-012-3526-5

Online publiziert: 10. Dezember 2012

c) Springer-Verlag Berlin Heidelberg 2012

K.E. Wartenberg ${ }^{1} \cdot$ M. Köhrmann ${ }^{2}$

${ }^{1}$ Klinik für Neurologie, Martin-Luther-Universität Halle-Wittenberg, Halle (Saale)

${ }^{2}$ Neurologische Universitätsklinik Erlangen

\title{
Intensivneurologie
}

ergebnis ist im Wesentlichen der initiale Schweregrad der Subarachnoidalblutung verantwortlich. Symptomatischer Vasospasmus beeinflusst das Outcome nur im Zusammenhang mit tatsächlich stattgehabter zerebraler Ischämie im Sinne der "delayed cerebral ischemia" (DCI). Multimodale Monitoringstudien erbrachten Hinweise auf multifaktorielle Ursachen und Therapieeffekte, z. B. das Konzept der „spreading depolarization“. Die Diagnostik stellt die Perfusionsbildgebung mehr in den Mittelpunkt und vom „Triple-H“Therapiekonzept ist nur die Hypertension effektiv in der Behandlung der DCI.

Die Bedeutung von nonkonvulsiven Status epileptici gerade in der neurologischen Intensivmedizin wird heiß diskutiert. Mit der zunehmenden Verfügbarkeit des kontinuierlichen EEG- und intrakortikalen Monitorings werden nonkonvulsive Anfälle sicherlich häufiger dokumentiert. Die klinische Signifikanz ist weniger gut untersucht. Es deutet sich aber an, dass ein nonkonvulsiver Status epilepticus in Abhängigkeit von der zugrunde liegenden Ursache oft mit einer ungünstigen Prognose vergesellschaftet ist. Inzwischen wird er in anderen Teilen der Welt aggressiv mit intravenösen Sedativa behandelt, die Datenlage ist nach wie vor begrenzt.

Multimodales Monitoring in der Neurointensivmedizin umfasst neben der Hirn- und Perfusionsdruckmessung, transkraniellem Dopplerultraschall und evozierten Potenzialen die intrazerebrale Messung von Hirnsauerstoffpartialdruck mittels Licox-Sonde, von Metaboliten und Neurotransmittern wie Glukose, Pyruvat, Laktat, Glutamat und Glyzerol mittels Mikrodialyse, die direkte Messung des zerebralen Blutflusses sowie kor- tikales oder Oberflächen-EEG-Monitoring. Durch den Einsatz dieses Monitorings wurden insbesondere Prozesse der Autoregulation bei zerebraler Hirnschädigung und Mechanismen der Sekundärschäden bei ischämischem Schlaganfall, Schädel-Hirn-Trauma, intrakraniellen Blutungen und anderen neurointensivmedizinischen Krankheitsbildern besser verstanden. Diese neuen Erkenntnisse sollten in zukünftige Behandlungsprotokolle einfließen. Ein Anfang ist die Brain Tissue Oxygen Monitoring in Traumatic Brain Injury (BOOST 2)-Studie, die die Effizienz von Behandlungsprotokollen zur Sicherstellung ausreichender zerebraler Oxygenierung testet.

Neue Therapieansätze bestimmen auch die Forschungslandschaft bei den intrazerebralen Blutungen. Mit dem intrazerebralen Blutvolumen als wichtigstem prognostischem Indikator gilt es, das Hämatomwachstum so schnell wie möglich einzudämmen oder das Hämatom in seiner Größe zu reduzieren. $\mathrm{Zu}$ diesen Behandlungszielen laufen zurzeit Phase-IIund -III-Studien zur Blutdrucksenkung nach intrazerebraler Blutung, Thrombolyse intraventrikulärer oder intrazerebraler Blutungen, Kraniotomie und stereotaktischen mikrochirurgischen Eingriffen zur Reduktion des Blutvolumens, Hypothermie sowie Ableitung von Blutabbauprodukten mittels lumbaler Drainage.

Im Sinne der Implementation von Leitlinien und evidenzbasierter Medizin in der Neurointensivmedizin, die in den vorherigen Arbeiten diskutiert werden, führten Mitglieder der IGNITE (Initiative for German NeuroIntensive Trial Engagement)-Gruppe eine Umfrage unter deutschen Neurointensivstationen zu deren Standards, Behandlungsprotokollen
Definition, Diagnostik und Therapie. Fur das funktionelle Langzeitbehandlungs- 
und Scores durch. Trotz zunehmender Veröffentlichung von Leitlinien werden diese in der täglichen Praxis nur teilweise angewandt. Anästhesiologisch geführte Neurointensivstationen nutzen die Therapiestandardisierung z. B. zur Hirndrucksenkung signifikant häufiger als neurologisch geführte Stationen. Die in Studien validierten Outcome-bestimmenden Scores werden eher selten im neurointensivmedizinischen Alltag berücksichtigt. Diese Tatsachen und die Beiträge dieses Heftes sollten uns also motivieren, die Situation und Ausbildung in der Neurointensivmedizin zu verändern.

Wir hoffen, dass Sie aus diesen Beiträgen Erkenntnisse für Ihr tägliches Management mitnehmen und wünschen Ihnen viel Spaß beim Lesen.

\section{Katji Wartemberg}

Dr. Katja Wartenberg

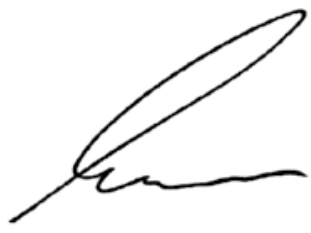

PD Dr. Martin Köhrmann

\section{Korrespondenzadresse}

\section{Dr. K.E. Wartenberg}

Klinik für Neurologie,

Martin-Luther-Universität Halle-Wittenberg

Ernst-Grube-Str. 40, 06120 Halle (Saale)

katja.wartenberg@medizin.uni-halle.de

\section{Ärzte Zeitung digital: Heute lesen, was morgen in der Zeitung steht}

\section{Im Publikumsbereich erfreuen sich App-Ausgaben von Zeitungen und Zeit- schriften wachsender Beliebtheit. Ab sofort ist auch Deutschlands einzige Ta- geszeitung für Ärzte in diesem Format erhältlich: die „Ärzte Zeitung digital“.}

„Ärzte Zeitung digital" bietet gesundheitspolitische Nachrichten, Kommentare, Hintergründe zu Medizin und wirtschaftlichen Themen rund um die Praxis - im übersichtlichen und intuitiven Lesemodus, optimiert für den Tablet-Computer. Die Vorteile für Leser der AppAusgabe der "Ärzte Zeitung":

- Die Ausgabe ist schon am Vorabend verfügbar, Leser können damit die Nachrichten des kommenden Tages bequem auf dem Sofa empfangen.

- Wichtige Nachrichten werden als „Breaking News" direkt auf das Tablet geleitet und lassen sich über die sogenannte Newslasche anzeigen.

- Die App ist so konzipiert, dass die gesamte Ausgabe im Hintergrund heruntergeladen werden kann. Anders als im Internet ist es für Leser dann möglich, sich offline durch die Ausgabe zu „blättern“, ohne Wartezeiten beim Durchklicken auf einer Website.

- Die alten Ausgaben bleiben zwei Wochen auf dem Tablet-PC verfügbar.

„Ärzte Zeitung digital“ ist Teil des e.Med-Pakets von Springer Medizin. Zugänglich ist die App über den iTunes-Store von Apple - allerdings nur für registrierte Nutzer von Springer Medizin, die e.Med für 30 Tage kostenlos testen wollen, oder für e.Med-Abonnenten - und zwar ohne weitere Zusatzkosten. Die Version für Android-Tablets wird Anfang 2013 verfügbar sein.

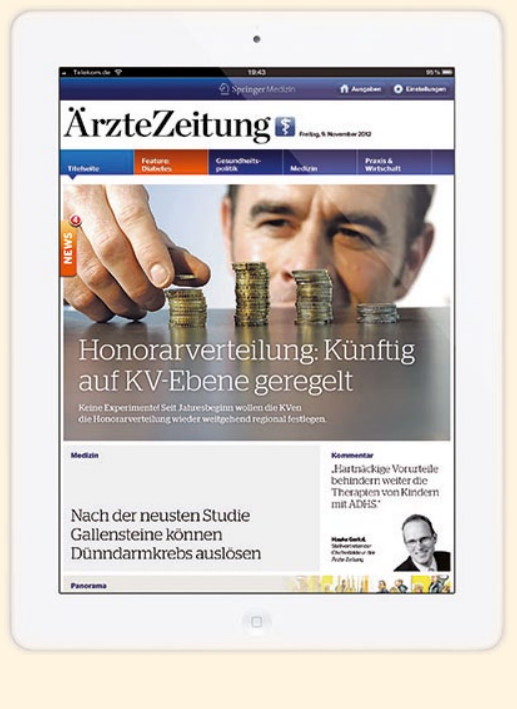

Abonnenten des e.Med-Pakets haben Zugriff auf rund 500 Fortbildungskurse in der e.Akademie, auf die Volltexte von mehr als 400 deutschsprachigen und internationalen Zeitschriften in der e.Bibliothek, auf einen Webbaukasten für die Praxiswebsite und ein PrintAbo einer Springer-Fachzeitschrift nach Wahl (plus Versandkostenpauschale). Das e.Med-Paket kostet 33,25 Euro im Monat.

Anmeldung zum kostenlosen e.Med-Testzugang: www.springermedizin.de/eMed/

Mehr Info zur App „Ärzte Zeitung digital“: www.springermedizin.de/tablet

Quelle: Ärzte Zeitung 